\title{
Relic Right-handed Dirac Neutrinos and Cosmic Neutrino Background
}

\author{
Shun Zhou ${ }^{* \dagger}$ \\ Institute of High Energy Physics, Chinese Academy of Sciences, Beijing 100049, China \\ Center for High Energy Physics, Peking University, Beijing 100871, China \\ E-mail: zhoush@ihep.ac.cn
}

The PTOLEMY experiment, implementing a $100 \mathrm{~g}$ surface-deposited tritium target, is promising to detect cosmic neutrino background via $v_{e}+{ }^{3} \mathrm{H} \rightarrow{ }^{3} \mathrm{He}+e^{-}$. In this talk, I consider a thermal production of right-handed Dirac neutrinos in the early Universe, and investigate their impact on the capture rate of cosmic relic neutrinos at PTOLEMY.

Neutrino Oscillation Workshop

4 - 11 September, 2016

Otranto (Lecce, Italy)

\footnotetext{
* Speaker.

${ }^{\dagger}$ This work was supported in part by the National Recruitment Program for Young Professionals and by the CAS Center for Excellence in Particle Physics (CCEPP).
} 


\section{Introduction}

One milestone achievement of the big bang cosmology is the prediction for cosmic microwave background $(\mathrm{CMB})$, which has now been precisely measured and led to a tremendous progress in our understanding of the Universe [1]. As another solid prediction from the big bang theory, cosmic neutrino background $(\mathrm{C} v \mathrm{~B})$ should exist as well and it must carry useful information about the early Universe when it was just one second old. Therefore, a direct detection of $\mathrm{C} v \mathrm{~B}$ in terrestrial laboratories is of crucial importance to test the standard cosmology on the one hand, and to open a new window on probing intrinsic properties of neutrinos themselves on the other hand.

When the temperature of the Universe dropped down to $T=T_{\mathrm{L}} \approx 1 \mathrm{MeV}$, the Hubble expansion rate exceeded the weak interaction rate of left-handed neutrinos $v_{\mathrm{L}}$ and right-handed antineutrinos $\bar{v}_{\mathrm{R}}$, and thus both $v_{\mathrm{L}}$ and $\bar{v}_{\mathrm{R}}$ decoupled from the thermal bath. At this moment, $v_{\mathrm{L}}$ and $\bar{v}_{\mathrm{R}}$ were extremely relativistic, given neutrino masses $m_{v} \lesssim 0.1 \mathrm{eV}$ [2]. Consequently, the number density $n_{v_{1}}$ of left-helical neutrinos $v_{1}$ was equal to that $n_{v_{\mathrm{L}}}$ of left-handed neutrinos $v_{\mathrm{L}}$, while the number density $n_{v_{\mathrm{r}}}$ of right-helical neutrinos $v_{\mathrm{r}}$ is vanishing. Hence we have $n_{v_{1}}=n_{v_{\mathrm{L}}}$ and $n_{v_{\mathrm{r}}}=0$ for neutrinos, while $n_{\bar{v}_{\mathrm{r}}}=n_{\bar{v}_{\mathrm{R}}}$ and $n_{\bar{v}_{1}}=0$ for antineutrinos, at the decoupling temperature $T_{\mathrm{L}}$. Since the helicity operator commutes with the free Hamiltonian, neutrino helicities after decoupling are always conserved in the rest frame of $\mathrm{C} v \mathrm{~B}$. As the Universe is expanding, the neutrino temperature will be red-shifted. Nowadays, the average temperature of CMB photons is $T_{\gamma}=2.725 \mathrm{~K}$, which is related to the neutrino temperature $T_{v}=(4 / 11)^{1 / 3} T_{\gamma} \approx 1.945 \mathrm{~K}$. The difference between $T_{\gamma}$ and $T_{v}$ can be traced back to the reheating of photons via $e^{+} e^{-} \rightarrow \gamma \gamma$ around $T=0.5 \mathrm{MeV}$. Therefore, we obtain average number densities $\bar{n}_{v_{1}}=\bar{n}_{\bar{v}_{\mathrm{r}}} \approx 56 \mathrm{~cm}^{-3}$ per neutrino flavor in the present Universe.

It is a great challenge to detect such low-energy relic neutrinos, whose average momentum is $\left\langle p_{v}\right\rangle \approx 5.28 \times 10^{-4} \mathrm{eV}$. One promising approach is to seize non-relativistic relic neutrinos by radioactive $\beta$-decaying nuclei [3], e.g., $v_{e}+{ }^{3} \mathrm{H} \rightarrow{ }^{3} \mathrm{He}+e^{-}$, for which there is no energy threshold of $v_{e}$. In this process, the signal is simply a peak located at a distance of $2 m_{v}$ from the endpoint of the $\beta$ spectrum for ${ }^{3} \mathrm{H} \rightarrow{ }^{3} \mathrm{He}+\bar{v}_{e}+e^{-}$[4]. The recently proposed PTOLEMY experiment will implement a $100 \mathrm{~g}$ surface-deposited tritium target and could reach an energy resolution of $0.15 \mathrm{eV}$, which will hopefully discover $\mathrm{C} v \mathrm{~B}$ [5]. See, e.g., Refs. [6, 7], for a review on this topic.

\section{Dirac Neutrinos}

The simplest extension of the Standard Model (SM) to accommodate tiny neutrino masses is to add three right-handed neutrino singlets and generate Dirac masses for neutrinos in the same way as for quarks and charged leptons. However, the huge hierarchy between neutrino masses $m_{v} \lesssim 0.1 \mathrm{eV}$ and top-quark mass $m_{t}=1.71 \times 10^{12} \mathrm{eV}$ needs to be further explained. Since the Yukawa couplings of Dirac neutrinos are extremely small $y_{v} \lesssim 10^{-12}$, the direct production of right-handed neutrinos $v_{\mathrm{R}}$ and left-handed antineutrinos $\bar{v}_{\mathrm{L}}$ in the early Universe is highly suppressed [8, 9]. Therefore, we have $n_{v_{\mathrm{r}}}=n_{\bar{v}_{1}}=0$ at $T=T_{\mathrm{L}}$ and today as well.

In Ref. [9], a working example has been given to thermally produce right-handed neutrinos $v_{R}$ and left-handed antineutrinos $\bar{v}_{\mathrm{L}}$. In this scenario, primordial magnetic fields $B_{0} \approx 10^{24} \mathrm{G}$ within a domain size $L_{0}>10^{-7} \mathrm{~cm}$ are assumed to be generated during the electroweak phase transition at $T=100 \mathrm{GeV}$. Although the evolution of such magnetic fields in the early Universe is not yet 


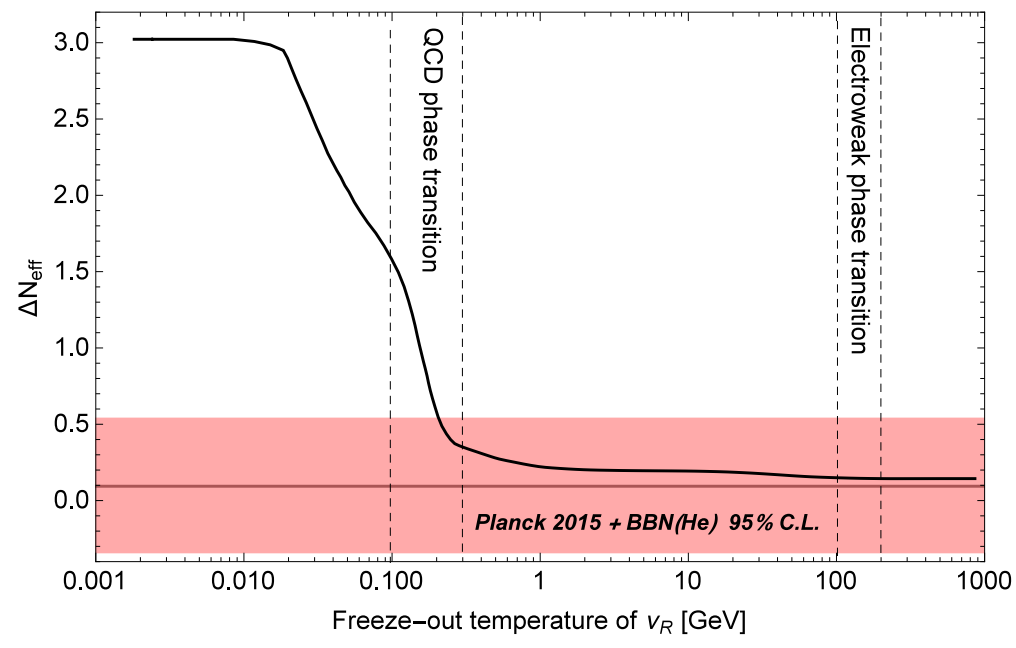

Figure 1: The extra effective number of neutrinos $\Delta N_{\text {eff }}$ is shown with respect to the decoupling temperature $T_{\mathrm{R}}$ of right-handed neutrinos [9].

quite clear, some phenomenological models are available [10]. It can be shown that massive Dirac neutrinos with a small magnetic dipole moment $\mu_{v}=3 \times 10^{-20}\left(m_{v} / 0.1 \mathrm{eV}\right) \mu_{\mathrm{B}}$, where $\mu_{\mathrm{B}}$ is the Bohr magneton, can experience spin-flipping conversions $v_{\mathrm{L}} \rightarrow v_{\mathrm{R}}$ and $\bar{v}_{\mathrm{R}} \rightarrow \bar{v}_{\mathrm{L}}$ in magnetic fields. For $B_{0} \approx 10^{24} \mathrm{G}$ and $L_{0}>10^{-7} \mathrm{~cm}$, these conversions are sufficiently rapid but become out of equilibrium in the epoch of QCD phase transition around $T \approx 200 \mathrm{MeV}$. As these additional thermal relics contribute to the total energy density just like ordinary neutrinos, they are subject to the cosmological upper bound on the extra effective number of neutrinos, namely, $\Delta N_{\text {eff }}<0.53$ at the $95 \%$ confidence level. In Fig. 1, one can observe that the decoupling temperature $T_{\mathrm{R}}$ of $v_{\mathrm{R}}$ and $\bar{v}_{\mathrm{L}}$ above $200 \mathrm{MeV}$ is compatible with the cosmological bound.

We should calculate the number densities of $v_{\mathrm{r}}$ and $\bar{v}_{1}$ at present by assuming that the upper bound $\Delta N_{\text {eff }}<0.53$ is saturated. First, it is straightforward to find the number density at $T_{\mathrm{L}}$ [9]

$$
\frac{n_{v_{\mathrm{r}}}\left(T_{\mathrm{L}}\right)}{n_{v_{1}}\left(T_{\mathrm{L}}\right)}=\frac{n_{v_{\mathrm{r}}}\left(T_{\mathrm{L}}\right)}{n_{v_{\mathrm{r}}}\left(T_{\mathrm{R}}\right)} \cdot \frac{n_{v_{1}}\left(T_{\mathrm{R}}\right)}{n_{v_{1}}\left(T_{\mathrm{L}}\right)}=\frac{g_{* \mathrm{~s}}\left(T_{\mathrm{L}}\right)}{g_{* \mathrm{~s}}\left(T_{\mathrm{R}}\right)},
$$

where $n_{v_{\mathrm{r}}}\left(T_{\mathrm{R}}\right)=n_{v_{1}}\left(T_{\mathrm{R}}\right)$ and $n_{v_{1}}\left(T_{\mathrm{R}}\right) / n_{v_{1}}\left(T_{\mathrm{L}}\right)=T_{\mathrm{R}}^{3} / T_{\mathrm{L}}^{3}$ hold for neutrinos in thermal equilibrium. For the decoupled $v_{\mathrm{R}}$ in the adiabatically expanding Universe, the entropy conservation gives rise to $n_{v_{\mathrm{r}}}\left(T_{\mathrm{R}}\right) / n_{v_{\mathrm{r}}}\left(T_{\mathrm{L}}\right)=\left[g_{* \mathrm{~s}}\left(T_{\mathrm{R}}\right) T_{\mathrm{R}}^{3}\right] /\left[g_{* \mathrm{~s}}\left(T_{\mathrm{L}}\right) T_{\mathrm{L}}^{3}\right]$, where $g_{* \mathrm{~s}}$ denotes the effective number of degrees of freedom contributing to the entropy density. Given $T_{\mathrm{R}} \approx 200 \mathrm{MeV}$ and $T_{\mathrm{L}} \approx 1 \mathrm{MeV}$, we get $g_{* \mathrm{~S}}\left(T_{\mathrm{R}}\right) \approx 38.4$ and $g_{* \mathrm{~s}}\left(T_{\mathrm{L}}\right) \approx 10.75$, implying that $n_{v_{\mathrm{r}}} / n_{v_{1}} \approx 28 \%$, which remains to be constant until today as both $v_{\mathrm{r}}$ and $v_{1}$ are decoupled below $T_{\mathrm{L}}$. Thus, the average number densities are $\bar{n}_{v_{\mathrm{r}}}=\bar{n}_{\bar{v}_{1}} \approx 16 \mathrm{~cm}^{-3}$ per neutrino flavor, which should be compared with $\bar{n}_{\bar{v}_{\mathrm{r}}}=\bar{n}_{\bar{v}_{1}} \approx 0$ in the case without thermal production of $v_{\mathrm{R}}$ and $\bar{v}_{\mathrm{L}}$.

\section{Capture Rates}

Now that the $\mathrm{C} v \mathrm{~B}$ is made of all four helical neutrino states, namely, $v_{1}$ and $\bar{v}_{\mathrm{r}}$ of an average number density $56 \mathrm{~cm}^{-3}$, and $v_{\mathrm{r}}$ and $\bar{v}_{1}$ of $16 \mathrm{~cm}^{-3}$, their capture rate on the tritium target should 
be changed. The capture rate for $v_{e}+{ }^{3} \mathrm{H} \rightarrow{ }^{3} \mathrm{He}+e^{-}$was first calculated in Ref. [11], and later corrected in Ref. [12]. Considering an unpolarized tritium target and a neutrino mass eigenstate $v_{i}$ of spin $s_{v}$ (i.e., $+1 / 2$ or $-1 / 2$ ), one can find that the product of the cross section $\sigma_{i}\left(s_{v}\right)$ and the neutrino velocity $v_{v_{i}}$ can be written as $\sigma_{i}\left(s_{v}\right) v_{v_{i}}=\mathscr{A}\left(s_{v}\right)\left|U_{e i}\right|^{2} \bar{\sigma}$, where $\bar{\sigma} \approx 3.8 \times 10^{-45} \mathrm{~cm}^{2}$, $\mathscr{A}\left(s_{v}\right) \equiv 1-2 s_{v} v_{v_{i}}$ and $U$ is the unitary lepton flavor mixing matrix. For non-relativistic $\mathrm{C} v \mathrm{~B}$ neutrinos with $v_{v_{i}} \rightarrow 0$, we have $\mathscr{A}(+1 / 2)=\mathscr{A}(-1 / 2) \approx 1$, implying that both left- and righthelical neutrino states can equally be captured [12]. The total capture rate is then given by

$$
\Gamma_{\mathrm{D}}=N_{\mathrm{T}} \sum_{i=1}^{3}\left[\sigma_{i}(-1 / 2) v_{v_{i}} \bar{n}_{v_{1}}+\sigma_{i}(+1 / 2) v_{v_{i}} \bar{n}_{v_{\mathrm{r}}}\right] \approx N_{\mathrm{T}} \bar{\sigma}\left(\bar{n}_{v_{1}}+\bar{n}_{v_{\mathrm{r}}}\right)
$$

where $N_{\mathrm{T}}$ is the number of tritium nuclei and the unitarity condition $\sum_{i}\left|U_{e i}\right|=1$ has been used. It is easy to observe that $\Gamma_{\mathrm{D}} \approx 4 \mathrm{yr}^{-1}$ in the standard case [12] will be increased to $\Gamma_{\mathrm{D}} \approx 5.1 \mathrm{yr}^{-1}$ in the presence of right-handed neutrinos in the early Universe [9]. As pointed out in Ref. [12], if massive neutrinos are Majorana particles, both $v_{1}$ and $\bar{v}_{\mathrm{r}}$ (now should be identified as $v_{\mathrm{r}}$ ) will participate in the capture process, leading to a twice larger rate $\Gamma_{\mathrm{M}} \approx 8 \mathrm{yr}^{-1}$.

A final remark is about further considerations on $\mathrm{C} v \mathrm{~B}$. In Ref. [13], a nonthermal production of $v_{\mathrm{R}}$ and $\bar{v}_{\mathrm{L}}$ from inflaton decays has been proposed for Dirac neutrinos. In this scenario, saturating the bound $\Delta N_{\text {eff }}<0.53$, the average number density $\bar{n}_{v_{\mathrm{r}}} \approx 29 \mathrm{~cm}^{-3}$ and thus a capture rate of $\Gamma_{\mathrm{D}} \approx 6.1 \mathrm{yr}^{-1}$ can be reached. Possible discrimination between thermal and nonthermal spectra of right-handed neutrinos may be achieved by observing the annual modulation at PTOLEMY [14].

\section{Acknowledgements}

I would like to thank Guo-yuan Huang and Jue Zhang for enjoyable collaboration on cosmic relic neutrinos, and the organisers of NOW 2016 for kind invitation and warm hospitality.

\section{References}

[1] S. Weinberg, Cosmology, Oxford University Press, 2008, Oxford, United Kingdom.

[2] C. Patrignani et al. (Particle Data Group), Chin. Phys. C 40, 100001 (2016).

[3] S. Weinberg, Phys. Rev. 128, 1457 (1962).

[4] J. M. Irvine and R. Humphreys, J. Phys. G 9, 847 (1983).

[5] S. Betts et al., arXiv:1307.4738.

[6] A. Ringwald, hep-ph/0505024.

[7] P. Vogel, AIP Conf. Proc. 1666, 140003 (2015).

[8] F. Antonelli, D. Fargion and R. Konoplich, Lett. Nuovo Cim. 32, 289 (1981).

[9] J. Zhang and S. Zhou, Nucl. Phys. B 903, 211 (2016) [arXiv:1509.02274].

[10] K. Enqvist, A. I. Rez and V. B. Semikoz, Nucl. Phys. B 436, 49 (1995) [hep-ph/9408255].

[11] A. G. Cocco, G. Mangano and M. Messina, JCAP 0706, 015 (2007) [hep-ph/0703075].

[12] A. J. Long, C. Lunardini and E. Sabancilar, JCAP 1408, 038 (2014) [arXiv:1405.7654].

[13] M. C. Chen, M. Ratz and A. Trautner, Phys. Rev. D 92, no. 12, 123006 (2015) [arXiv:1509.00481].

[14] G. Y. Huang and S. Zhou, arXiv:1610.01347. 